\title{
Incidental Small Bowel Adenocarcinoma Upon Surgical Evaluation of a Retained Endoscopic Video Capsule in a Patient With Crohn's Disease
}

\author{
Mahmoud Aryan ${ }^{\mathrm{a}, \mathrm{c}} \mathbb{0}$, Nabeel Moon ${ }^{\mathrm{b}}$
}

\begin{abstract}
A 28-year-old male with Crohn's disease presented with hematochezia and severe anemia. Upon evaluation, no source of bleeding was identified with colonoscopy or esophagogastroduodenoscopy; therefore, a video capsule was placed. Following capsule placement, the patient developed severe abdominal pain. Abdominal X-ray revealed the capsule at the ileocecal valve, indicating it was possibly stuck in a stricture. To reduce inflammation, intravenous steroids were started to assist with capsule passage. Following unsuccessful passage and continual abdominal pain, surgical investigation visualized a mass arising from a jejunal Crohn's stricture. Despite multiple inspections of the resected small bowel, the capsule was not located. Intraoperative $\mathrm{X}$-ray revealed the capsule inside the large bowel, indicating capsule passage through the stricture. Final pathology revealed metastatic poorly differentiated adenocarcinoma. This case demonstrates management of a retained capsule in Crohn's patients and emphasizes the importance of considering small bowel adenocarcinoma in Crohn's patients with obstructive signs.
\end{abstract}

Keywords: Crohn's disease; Small bowel; Cancer; Steroids; Capsule; Endoscopy

\section{Introduction}

Small bowel adenocarcinomas represent $1-5 \%$ of gastrointestinal tract malignancies; however, in patients with Crohn's disease as compared to the general population, there is over a 60 -fold increased risk of small bowel adenocarcinoma [1].

Manuscript submitted April 27, 2021, accepted June 5, 2021

Published online June 19, 2021

aDepartment of Internal Medicine, University of Alabama at Birmingham, Birmingham, AL 35233, USA

bepartment of Internal Medicine, University of Florida College of Medicine, Gainesville, FL 32610, USA

${ }^{\mathrm{c} C}$ Corresponding Author: Mahmoud Aryan, Department of Internal Medicine, University of Alabama at Birmingham, 1720 2nd Ave S, Birmingham, AL 35294, USA. Email: mahmoudaaryan@gmail.com

doi: https://doi.org/10.14740/gr1403
Small bowel adenocarcinoma is a recognized complication of Crohn's disease [2]. Inflammation in the small bowel serves as a nidus for cancer development. Further risk factors that predispose Crohn's disease patients to develop cancer include male sex, chronic fistulous disease, and loops of small bowel that are bypassed following surgery [3]. Adenocarcinoma often develops in the small bowel segments that are involved in Crohn's disease as well as within strictures [2, 4]. Obstructive symptoms are typically the primary symptoms that present in such patients. Other symptoms that may occur as a result of small bowel adenocarcinoma such as bleeding, pain, and bloating are often presumed to be due to a Crohn's flare. Diagnostic imaging such as computed tomography (CT) and magnetic resonance imaging (MRI) may miss small lesions and often are unable to differentiate between small bowel adenocarcinoma and severe Crohn's disease [2]. Seventy percent of small bowel adenocarcinoma in Crohn's patients is found incidentally on pathological examination of resected specimens [2]. While colonoscopies serve as a screening tool for colon cancer in Crohn's disease, screening of small bowel cancer remains a challenge. Prognosis is also extremely poor. Studies have shown up to $72 \%$ mortality with a mean survival of only 7.9 months from the diagnosis of cancer [5]. Two year survival rates have been found to be as low as $27 \%$ [6]. Favorable prognosticators included jejunal location, absence of nodal metastases, and a well-differentiated grade [7]. While radiotherapy and chemotherapy for small bowel adenocarcinoma are primarily palliative measures, surgery can be curative [8]. Wide local excision with lymphadenectomy is the procedure of choice [8]. Here we report the case of a patient in whom a video capsule endoscopy got lodged in a small bowel stricture. Following evacuation of the capsule, small bowel adenocarcinoma was incidentally found.

\section{Case Report}

A 28-year-old male with a history celiac disease, chronic iron deficiency anemia, and Crohn's disease diagnosed 15 years prior to presentation and on biweekly adalimumab presented for evaluation of anemia and intermittent bright red blood per rectum. Over the span of 4 months, he had noticed several milliliters of blood at the end of each bowel movement in the toilet and had felt progressively more fatigued. The pa- 


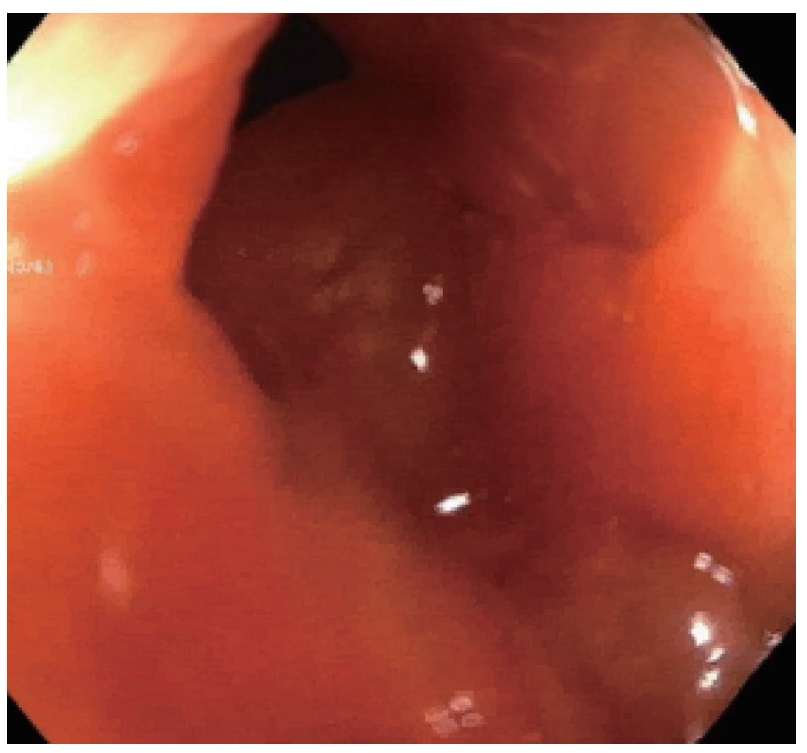

Figure 1. Colonoscopy image of the stenotic ileocecal valve.

tient denied any diarrhea, abdominal pain, rectal pain, recent trauma, or any other areas of bleeding. The patient had previously been on budesonide but was escalated to adalimumab following persistent Crohn's symptoms. His last colonoscopy was 1 year ago with findings consistent with Crohn's disease in the ileum and cecum, but no other areas of colonic or small bowel involvement were seen. Additionally, no arteriovenous malformations, fistulizing disease, masses, polyps, or signs of bleeding were visualized. The patient had no extraintestinal manifestations of Crohn's disease, and his disease was overall well controlled with no flares within the previous 2 years.

On examination in the emergency department, he was afebrile with a blood pressure of $110 / 65 \mathrm{~mm} \mathrm{Hg}$, heart rate of $87 / \mathrm{min}$, and respiratory rate of $16 / \mathrm{min}$. Physical exam was remarkable for pale conjunctiva bilaterally, benign abdominal exam, and rectal exam revealed blood tinged stool present in the rectal vault, intact sphincter tone, and no abnormal masses. Hemoccult test was positive, and the complete blood count revealed a hemoglobin level of 4 . His C-reactive peptide (CRP) was $0.36 \mathrm{mg} / \mathrm{L}$ (reference: $0-5$ ), and his erythrocyte sedimentation rate (ESR) was $7 \mathrm{~mm} / \mathrm{h}$ (reference: 0 - 0). He was given two units of packed red blood cells and admitted to the inpatient gastroenterology service.

\section{Investigations}

Workup began with CT imaging of the abdomen and pelvis with contrast revealing multiple distal loops of small bowel with areas of wall thickening and luminal hyperemia consistent with inflammatory enteritis. No evidence of active gastrointestinal bleeding was seen.

A colonoscopy was then preformed which revealed stenosis of the ileocecal valve characterized by inflammation with erosions, erythema, friability and aphthous ulcerations (Fig. 1). The entire examined segment of the colon appeared nor-

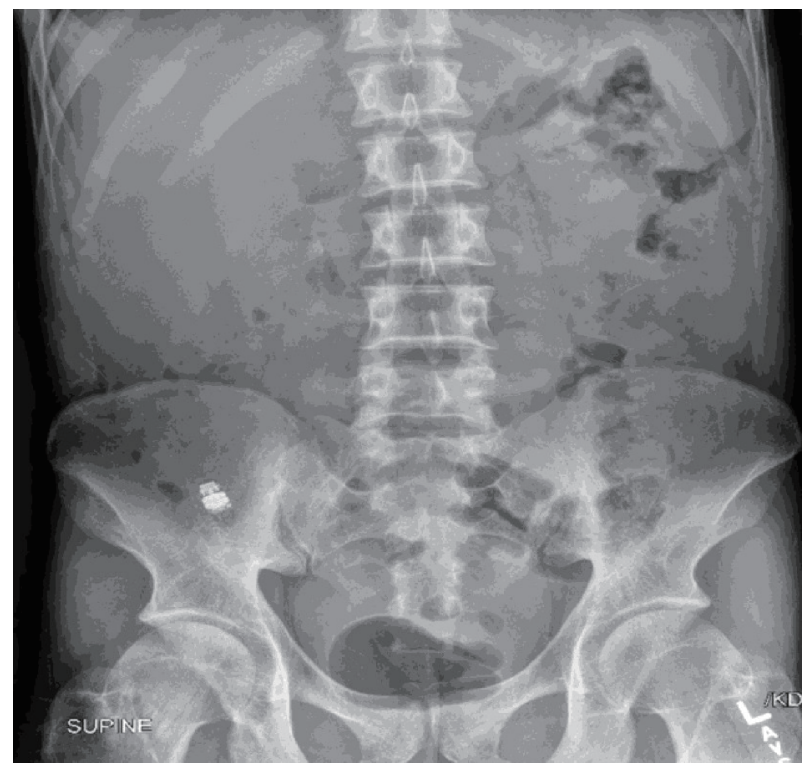

Figure 2. Abdominal X-ray demonstrating the endoscopy capsule in the ileocecal valve.

mal. The colonoscope was not able to traverse the ileocecal valve. Biopsies of the stenotic ileocecal valve were taken and showed mild chronic active ileitis with no signs of granulomas or dysplasia. Biopsies of the left colon, right colon, and rectum depicted normal mucosa.

Esophagogastroduodenoscopy (EGD) revealed no upper tract pathology, and a video capsule enteroscope was placed during the EGD. The capsule revealed a non-bleeding jejunal ulcer, nodular mucosa in a cobble stone pattern, non-bleeding angiodysplastic lesions, and a plume of blood entering the lumen at $3 \mathrm{~h}$ and $42 \mathrm{~min}$. The following day the patient began having severe abdominal pain, and the capsule had not passed yet. An abdominal X-ray revealed that the capsule was still at the ileocecal valve, likely indicating that it was stuck at a stricture (Fig. 2).

The patient was given one dose of $60 \mathrm{mg}$ of intravenous methylprednisolone in an attempt to reduce inflammation to allow for passage of capsule. This was then transitioned to $60 \mathrm{mg}$ of prednisone daily for 1 week followed by a taper of $10 \mathrm{mg}$ per week. The capsule continued to cause pain despite steroid treatment, and colorectal surgery was consulted. Upon evaluation, it was determined that surgical intervention would be necessary to remove the capsule as well as possible removal of the stricture.

\section{Diagnosis}

Upon initial presentation with a 4-month history of bright red blood per rectum in a patient with Crohn's disease, the differential was broad and included active Crohn's inflammation, arteriovenous malformations, deep bleeding ulcer, hemorrhoids, diverticulosis, and malignancy. Following a non-revealing upper and lower endoscopy as well the development of abdominal pain with retention of video capsule endoscopy, the dif- 


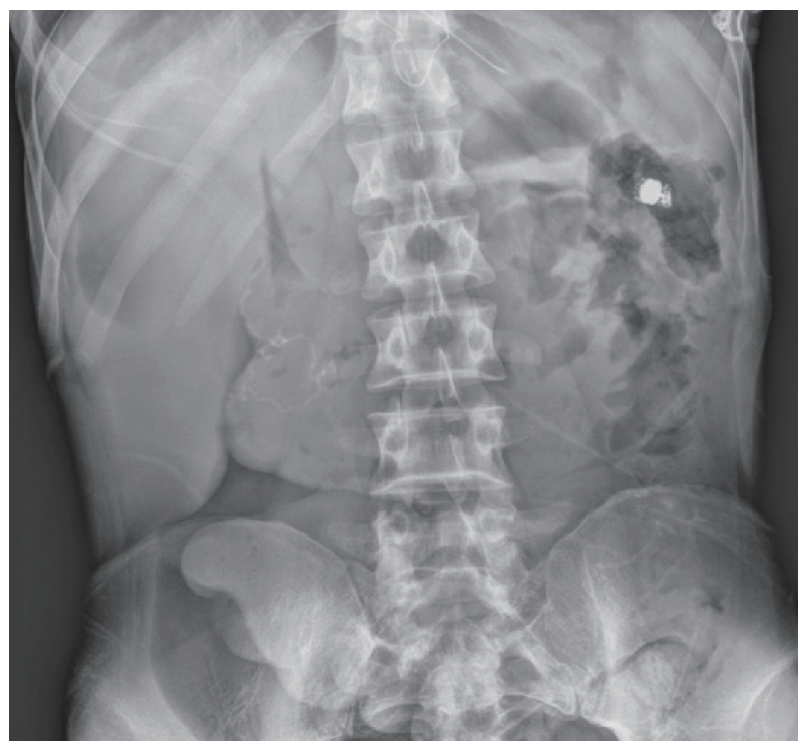

Figure 3. Abdominal X-ray demonstrating the endoscopy capsule in the splenic flexure.

ferential was modified given the patient's signs of obstruction. With the patient's history of Crohn's disease, the small bowel may be subject to several different types of insults including strictures, fistulas, ulcers, and even malignancies. All of these possibilities were included within our differential, but given the urgency of such retained capsule in a clinically decompensating patient, surgical intervention was deemed necessary to determine the etiology.

\section{Treatment}

A laparoscopic exploration of the small bowel was performed. In the operating room the surgeons visualized a tumor in the jejunum arising from a Crohn's stricture with diffuse carcinomatosis as well as multifocal Crohn's disease involving the terminal ileum. The jejunal tumor, significant strictures, terminal ileum, and ascending colon were resected. One of the peritoneal nodules in the pelvis was also biopsied. Interestingly, despite multiple inspections of the resected small bowel, the capsule could not be located. An intraoperative X-ray revealed the capsule in the left upper quadrant in what appeared to be the splenic flexure region of the colon (Fig. 3). The area was reinspected laparoscopically, and due to the stool burden in the colon, the capsule still could not be found. Given the fact the capsule was definitively not in the small bowel, it was concluded that the capsule passed through the stricture prior to resection.

Since previous colonoscopy did not reveal any large bowel obstruction, there was assurance that the capsule would pass spontaneously. An abdominal X-ray done on postoperative day 4 showed no capsule.

The final pathology of the jejunal resection, terminal ileal resection, and peritoneal biopsy revealed metastatic poorly differentiated adenocarcinoma with signet ring cell and mucinous features involving the ileum, appendix, peritoneum, and mes-

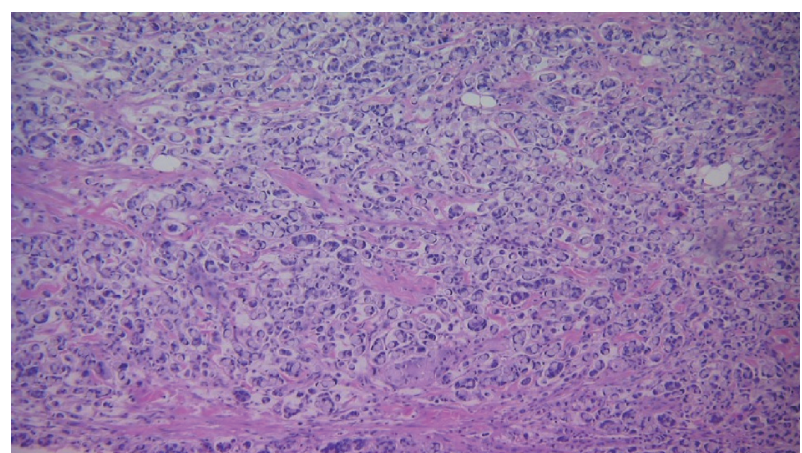

Figure 4. Jejunal tumor biopsy indicating poorly differentiated adenocarcinoma with signet ring cell and mucinous features.

entery (Fig. 4). The pathological staging was pT4N1M1. There was intact mismatch repair protein (MLH1, PMS2, MSH2, MSH6) nuclear expression.

\section{Follow-up and outcomes}

The patient's postoperative course was complicated by pelvic abscess formation which required interventional radiology drain placement and antibiotic treatment. He was discharged on postoperative day 11 . He followed in clinic 12 days after and was feeling well, eating well, with no obstructive symptoms. He was referred to a tertiary oncological center for further care.

\section{Discussion}

Although small bowel adenocarcinoma is a recognized complication of Crohn's disease, it is often overlooked. In patients presenting with obstructive symptoms and severe anemia in the setting of non-concerning colonoscopies such as in our patient, small bowel adenocarcinoma should be strongly considered. While imaging modalities such as CT scans are often low yield in diagnosing small bowel adenocarcinoma, non-responsiveness of symptoms to Crohn's treatment should prompt consideration of small bowel adenocarcinoma rather than treatment failure. The decision for surgery is never a light one; however, in severe cases surgery may be necessary. Surgical intervention in our patient allowed proper diagnosis as well as a pathway for further care.

While the inability of the capsule to pass in our patient was thought to be due to it being lodged in a stricture, small bowel obstruction due to small bowel adenocarcinoma should have also been included in the differential. The capsule was indeed lodged in a stricture in which the adenocarcinoma had invaded. Capsule retention occurs in 1-2\% of cases and is diagnosed via abdominal X-ray or CT scan such as in our case [9, 10]. A capsule is expected to reach the colon in $8 \mathrm{~h}$, pass in the stool within $48 \mathrm{~h}$, and often is undetected by the patient [10, 11]. A retained capsule often remains asymptomatic; however, the patient is at risk of perforation, secondary obstruction, or ulceration $[12,13]$. The risks should not undermine the role of 
capsule endoscopy in Crohn's disease as studies have shown capsule endoscopy to more accurately determine the severity and extent of Crohn's disease in the small bowel over traditional imaging modalities [14]. Double balloon enteroscopy is considered a primary method for retained capsule retrieval [15]. The most frequently used method however is surgical exploration [9]. With our patient, the concern for the capsule being lodged in a stricture made surgery the best option.

Prior to surgical intervention, we administered one dose of $60 \mathrm{mg}$ intravenous methylprednisolone in order to reduce inflammation in an attempt to allow passage of the capsule. In Crohn's disease patients, immunosuppressive medications such as steroids and infliximab have been shown to facilitate passage of the capsule and in some cases have prevented the need for surgery [16]. In the 5 day interval between the first abdominal X-ray and the intraoperative X-ray, the capsule appeared to have passed beyond the stricture. Given that the capsule was in the colon in the intraoperative image, the capsule likely passed shortly before the surgery. This case demonstrates that immunosuppressive medications may have a role in facilitating the passage of endoscopic capsules. Although immunosuppressive drugs may not prevent surgery, they may allow for a delay in surgery or a relief of symptoms.

\section{Learning points}

Small bowel adenocarcinoma should be considered in patients with Crohn's disease who present with obstructive symptoms. Prolonged retention of endoscopic capsules may lead to serious complications and therefore must be acted on in a timely and effective manner. Immunosuppressive medication may have a role in aiding passage of endoscopic capsules in patients with Crohn's disease.

\section{Acknowledgments}

None to declare.

\section{Financial Disclosure}

None of the authors have relevant disclosures, and this research did not receive any specific grant from funding agencies in the public, commercial, or not-for-profit sectors.

\section{Conflict of Interest}

None of the authors have relevant conflict of interest or competing interest to declare related to this study.

\section{Informed Consent}

Informed patient consent was obtained for the publication of this article.

\section{Author Contributions}

M. Aryan was involved in obtaining informed consent. All authors were involved in clinical managements as well as indepth literature review. All authors were involved in manuscript writing, approved the final manuscript, and take full responsibility for the manuscript.

\section{Data Availability}

The authors declare that data supporting the findings of this study are available within the article.

\section{Abbreviations}

CT: computed tomography; EGD: esophagogastroduodenoscopy

\section{References}

1. Jess T, Winther KV, Munkholm P, Langholz E, Binder V. Intestinal and extra-intestinal cancer in Crohn's disease: follow-up of a population-based cohort in Copenhagen County, Denmark. Aliment Pharmacol Ther. 2004;19(3):287293.

2. Cahill C, Gordon PH, Petrucci A, Boutros M. Small bowel adenocarcinoma and Crohn's disease: any further ahead than 50 years ago? World J Gastroenterol. 2014; 20(33):11486-11495.

3. Lashner BA. Risk factors for small bowel cancer in Crohn's disease. Dig Dis Sci. 1992;37(8):1179-1184.

4. Christodoulou D, Skopelitou AS, Katsanos KH, Katsios C, Agnantis N, Price A, Kappas A, et al. Small bowel adenocarcinoma presenting as a first manifestation of Crohn's disease: report of a case, and a literature review. Eur J Gastroenterol Hepatol. 2002;14(7):805-810.

5. Hawker PC, Gyde SN, Thompson H, Allan RN. Adenocarcinoma of the small intestine complicating Crohn's disease. Gut. 1982;23(3):188-193.

6. Dossett LA, White LM, Welch DC, Herline AJ, Muldoon RL, Schwartz DA, Wise PE. Small bowel adenocarcinoma complicating Crohn's disease: case series and review of the literature. Am Surg. 2007;73(11):1181-1187.

7. Ouriel K, Adams JT. Adenocarcinoma of the small intestine. Am J Surg. 1984;147(1):66-71.

8. Neugut AI, Marvin MR, Chabot JA. Adenocarcinoma of the small bowel. In: Surgical treatment: evidence-based and problem-oriented. 2001. Zuckschwerdt.

9. Liao Z, Gao R, Xu C, Li ZS. Indications and detection, completion, and retention rates of small-bowel capsule endoscopy: a systematic review. Gastrointest Endosc. 2010;71(2):280-286.

10. Cave D, Legnani P, de Franchis R, Lewis BS. ICCE consensus for capsule retention. Endoscopy. 2005;37(10):10651067. 
11. Srai R, Tullie L, Wadoodi A, Saunders M. Capsule endoscopy: a dangerous but diagnostic tool. BMJ Case Rep. 2013;2013:bcr-2013-009932.

12. Li F, Gurudu SR, De Petris G, Sharma VK, ShiffAD, Heigh RI, Fleischer DE, et al. Retention of the capsule endoscope: a single-center experience of 1000 capsule endoscopy procedures. Gastrointest Endosc. 2008;68(1):174-180.

13. Ali A, Santisi JM, Vargo J. Video capsule endoscopy: a voyage beyond the end of the scope. Cleve Clin J Med. 2004;71(5):415-425.

14. O'Donnell S, Qasim A, Ryan BM, O'Connor HJ, Breslin
$\mathrm{N}, \mathrm{CA}$ OM. The role of capsule endoscopy in small bowel Crohn's disease. J Crohns Colitis. 2009;3(4):282-286.

15. Van Weyenberg SJ, Van Turenhout ST, Bouma G, Van Waesberghe JH, Van der Peet DL, Mulder CJ, Jacobs MA. Double-balloon endoscopy as the primary method for small-bowel video capsule endoscope retrieval. Gastrointest Endosc. 2010;71(3):535-541.

16. Vanfleteren L, van der Schaar P, Goedhard J. Ileus related to wireless capsule retention in suspected Crohn's disease: emergency surgery obviated by early pharmacological treatment. Endoscopy. 2009;41(Suppl 2):E134-135. 